\title{
Challenges in the design of pilot tests
}

\author{
S. Barrera ARCADIS, Chile \\ M. Becerra ASMIN Industrial Ltda., Chile \\ P. Chacón ARCADIS, Chile \\ H. Quelopana ARCADIS, Chile
}

\begin{abstract}
The behaviour of tailings slurries, particularly at high concentrations, presents a significant number of uncertainties. To address this issue, it is common practice to perform pilot tests to verify or anticipate the expected tailings behaviour at large scale. In the last year three pilot tests were conducted on different kinds of tailings and conditions. The scaling and implementation of these tests present challenges derived mainly from the nature of a material formed by two phases and its tendency for sedimentation, the viscous behaviour of the fluid, the presence and variation in time of chemical additives, representativeness of tested material, differences in consultants/owners conceptual approach, etc.
\end{abstract}

The pilot tests need to be designed in such a way that allows us to obtain key information and extrapolate this information to our real case. To achieve this, it is at least necessary to address the following types of definitions: (i) parameters to be analysed; (ii) conceptual model; (iii) tests specifications; (iv) model scale; (v) type of material and concentration levels to be tested; (vi) type and amount of material; and (vii) available equipment; among others.

This paper describes the process in the design and start-up of a pilot plant oriented at studying the behaviour of a slimes slurry, highlighting the difficulties encountered and the ways of addressing and overcoming them. This paper does not analyse the test results, analysis which will be part of another document.

\section{Introduction}

The technology of tailings and paste, which initially was conceived to reduce the size of the containment dams, has become one of the most promising technologies to reduce water consumption in tailings management and facilitate the closing of operations. This technology has meant delving into unknown areas or areas of limited knowledge with regards to tailings behaviour, requiring the application of new theories and performing special testing to determine the associated unknowns. One way of overcoming these limitations is by performing tests at a larger scale than laboratory tests (also known as 'pilot tests') where the variables of interest are measured and evaluated. These tests are required in three scenarios in which:

- the current knowledge and/or tools available are exceeded

- the verification of a theoretical model is required

- the bounding of the uncertainty in the design variables is sought.

The uncertainty of knowledge of the behaviour of the variables of a system or model may affect the design in aspects such as:

- a longer execution time

- the need to provide flexibility to overcome unexpected behaviour changes

- and/or the under or over design of the system.

These tests are an option to significantly reduce these effects. 
Pilot tests face two types of challenges that are of a very different nature but which may significantly affect the results. The first of these refers to the representativeness of the material to be tested. The second is the definition of the scale and characteristics of the tests that allows us to make reliable extrapolations for full scale applications. It is worth noting that there are no standard rheological tests for tailings (this does not happen in geotechnical area). Both of these challenges are addressed in this paper where we have used our experience of the last campaigns with different types of tailings.

\section{$2 \quad$ Key parameters in the thickened tailings design}

The process of tailings thickening is an area that improves day to day and in which providers of thickening equipment have plans and has set aside resources for research and development. However, all the aspects of tailings management after thickening - from the thickener discharge - are an area with limited current knowledge: transport and deposition. In this manner, the planning, design and operation of a pilot plant are activities that take on great importance in the search for improving and optimising the engineering of a particular project.

In the area of transport of thickened tailings, the following subjects to be analysed and implemented are identified:

1. Discharge of the tailings thickeners at a high concentration in weight: In some cases, the discharge and subsequent transport of the tailings from the thickener is difficult due to the high yield stress derived from the presence of the flocculent and the intrinsic features of the tailings. To reduce the yield stress without lowering the concentration of the tailings is usual to generate a process of high turbulence or agitation that causes the destruction of flocs. This is normally done with centrifugal pumps and the process is called shear thinning. In consequence, the pilot plant is useful to analyse options to reduce the tailings yield stress and measure the effect at different concentrations in weight both at the discharge of the thickener as well as at the discharge of the solution (design) being evaluated.

2. Transport of thickened tailings: Depending on the tailings rheology and the project conditions (topography, production rate, etc.), the tailings transport may be limited due to lack of geometric height (friction losses) or the possibility of sedimentation of the tailings (in longer transport lines) or excessive wear. The need to have pumps, the type of pumps, the type of transport (open or under pressure flow), the size of the transport line (section or diameter), among other aspects, must be resolved for the project. Although there are hydraulic models to evaluate these transport issues, the pilot plant provides additional information with regards to velocity, effective head loss, wear, and grain size changes of the behaviour of the tailings slurry that is useful for the verification and the improvement of some model parameters.

3. Deposition of thickened tailings: The estimate of the deposition slope of tailings is a key parameter in the disposal scheme of an impoundment, allowing us to determine the discharge points, the height of the containment dam, the elevations of the civil works in the impoundment (spillways, roads, intake towers, etc.), the areas under tailings, etc. Currently, there isn't a single method to estimate deposition slope of a tailings but rather different approaches to the problem: theoretical, experimental and theoretical-experimental. To the above, we must add the fact that the beach slope is not a linear function of the main parameters and that experimentation in laboratory (at a small scale) is of little relevance. The proposed models require pilot scale tests to calibrate the parameters and allow their extrapolation to a real case. In this case, the tests are essential and unavoidable.

For each one of these subjects, tests are defined or specified in accordance with material features, objectives and availability of equipment, facilities, professional and technical staff, power, water, and air.

\section{Representativeness of the material}

The successful execution of the pilot tests requires, in the first place, the availability in quality and quantity of representative tailings material that will effectively be produced during the useful life of the mine. In this aspect, it may be considered to be outside of the testing but, in the authors' opinion, forms an inseparable part of a comprehensive focus in the design of the tailings management facilities. 
The representativeness of the material must be understood in this case as being related with the tailings behaviour in transport and deposition or, in other terms, mainly with rheology. In this case, the mineralogical or metallurgical characteristics are not relevant since what is looked for are samples that represent average and extreme values of the material in its transport and deposition.

The most complex case of representativeness is presented in a new project where there isn't a concentrator plant that processes the material and, therefore, there are no 'real' tailings. In these cases, there are limited amounts of tailings coming from pilot plants with a metallurgical objective and, in less common cases, we have tailings from other operations with similar characteristics.

In all cases, a characterisation methodology is applied to the different types of tailings in the mine with the objective of quantifying the main rheological and deposition parameters and, in this manner, identifies or group their behaviour ranges and finally select the critical tailings for design. The next step is to prepare tailings with similar properties to the ones selected. For this step, there are several options. In some cases, additional quantities of the ore that originated the critical sample are processed. In other cases, the solution is more elaborate since there is the need to combine tailings from different sources to prepare the material with similar characteristics to the critical tailings. In this last case, the characterisation and homogenising of the material become key elements in generating the representative sample.

It is worth noting that the emphasis in this step is in the imperative need of having adequate representativeness of the material to be tested more than in providing methodologies to resolve the problem. We understand that in this subject, there are many ways of doing it and that there isn't a one and only solution but rather that there are diverse solutions or approaches.

\section{$4 \quad$ Main challenges}

\subsection{General aspects}

Whichever test is being performed, a key aspect is the definition of the characteristics. This may be obvious but it is not so in many cases of tailings analysis. For example, an objective may be the assessment of the friction losses of a flow. But this test also requires determining if some level of sedimentation is acceptable or not. This implies adopting a model that includes the restrictions of sedimentation and its extrapolation to a real case.

In this sense, it is worth highlighting what was stated by Calle et al. (2009), "the design of industrial facilities with Bingham-type flows, such as those of high density tailings, is complex and requires in many cases the construction of physical models. Experience indicates that to avoid laminarisation in these tailings slurry, the transport of this fluid must be in turbulent regime, which implies an additional restriction in the problem of scaling" (translated by the authors).

Another important aspect is assuring that the testing material keeps its intrinsic properties during testing. That is, that the grain size distribution does not suffer significant changes. In general, these changes are not expected in metallic tailings but it is good practice to verify the gradation before and after the tests.

The previous metallurgical and rheological characterisation of the tailings sample to be tested in the pilot tests, especially high values of yield stress, provides indications of the size and strength of the equipment to be used with regards to power, motor, reducers, agitators, tank size, type of pump, etc. Once the above aspects are considered, the tests to be performed are specified and the equipment for each case is constructed and installed.

\subsection{Shear thinning tests}

As mentioned above, the high yield stress values obtained in highly thickened tailings - in some cases up to $600 \mathrm{pa}$ - cause difficulties in the discharge of the thickener. The continuous 'shear thinning' pilot test was designed to determine the response of these tailings to the mechanical agitation produced by an agitator or centrifugal pump. 
This pilot test consists of installing a tank with a variable speed agitator and power measurements at the discharge of one of the pilot thickeners and measuring the concentration of solids and yield stress in the following locations:

- before the pump discharging the pilot thickener

- after the pump discharging the pilot thickener, before the agitation tank

- discharge of the agitation tank, before the discharge to the disposal

- after the tailings disposal pump

- final tailings discharge.

\section{SHEAR THINNING PILOT TEST}

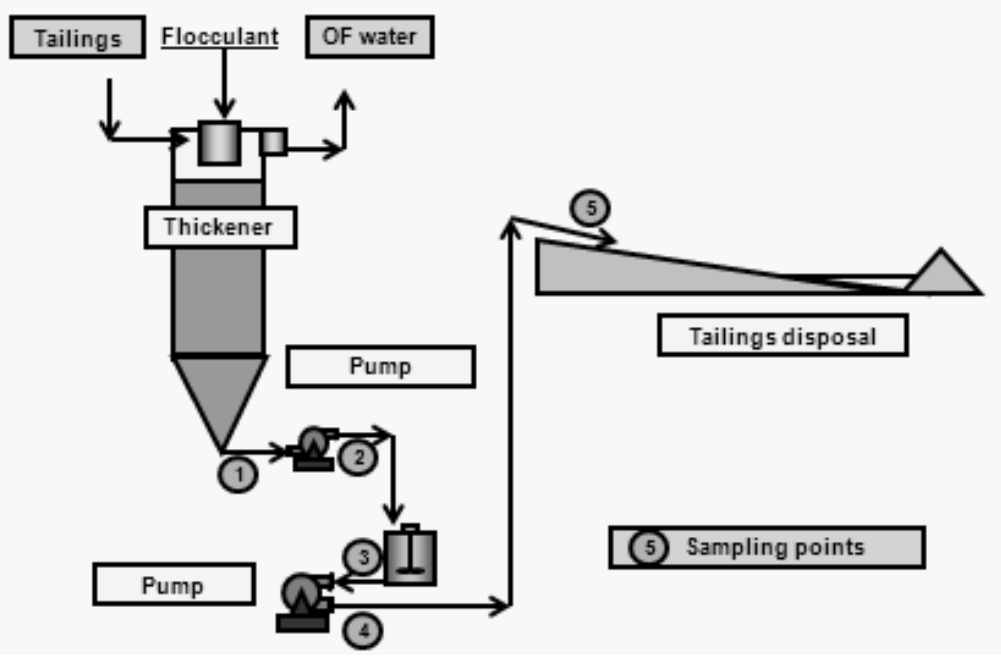

\section{Figure 1 Shear thinning pilot test diagram}

The challenges of this test are focused on assuring that the flow of flocculated tailings of the thickener be continuous to the shear thinning equipment (agitator or centrifugal pump). It is our opinion that it is not admissible to perform this type of test with material that does not come directly from the thickener (only way to ensure an equivalent material). In addition, it is relevant that the operation of the equipment be comparable to the equipment that will be used for operations. This means for instance that the speed of the impellers be similar as well as other relevant aspects.

\subsection{Transport tests}

The transport tests are usually done in a closed circuit (loop) to minimise the volume of tailings and obtain a greater number of measurements. In this mode, the tailings are reused many times making it necessary to verify its integrity. It is obvious that those characteristics that are sensitive to agitation and/or duration (yield stress by flocculation) may only be measured at the start since its effect will be significantly reduced throughout time.

There are two types of tests: open flow; and under pressure flow. What follows are the characteristics of both types.

\subsubsection{Open flow transport tests}

The open flow transport test is done in a pumping loop consisting of an elevated tank that feeds an adjustable inclined pipe that in turn discharges to another tank. This tank feeds a pump that sends the tailings back to the elevated tank, closing the loop. Figure 2 shows a schematic diagram of the main equipment used in the test and the sampling point's locations. 


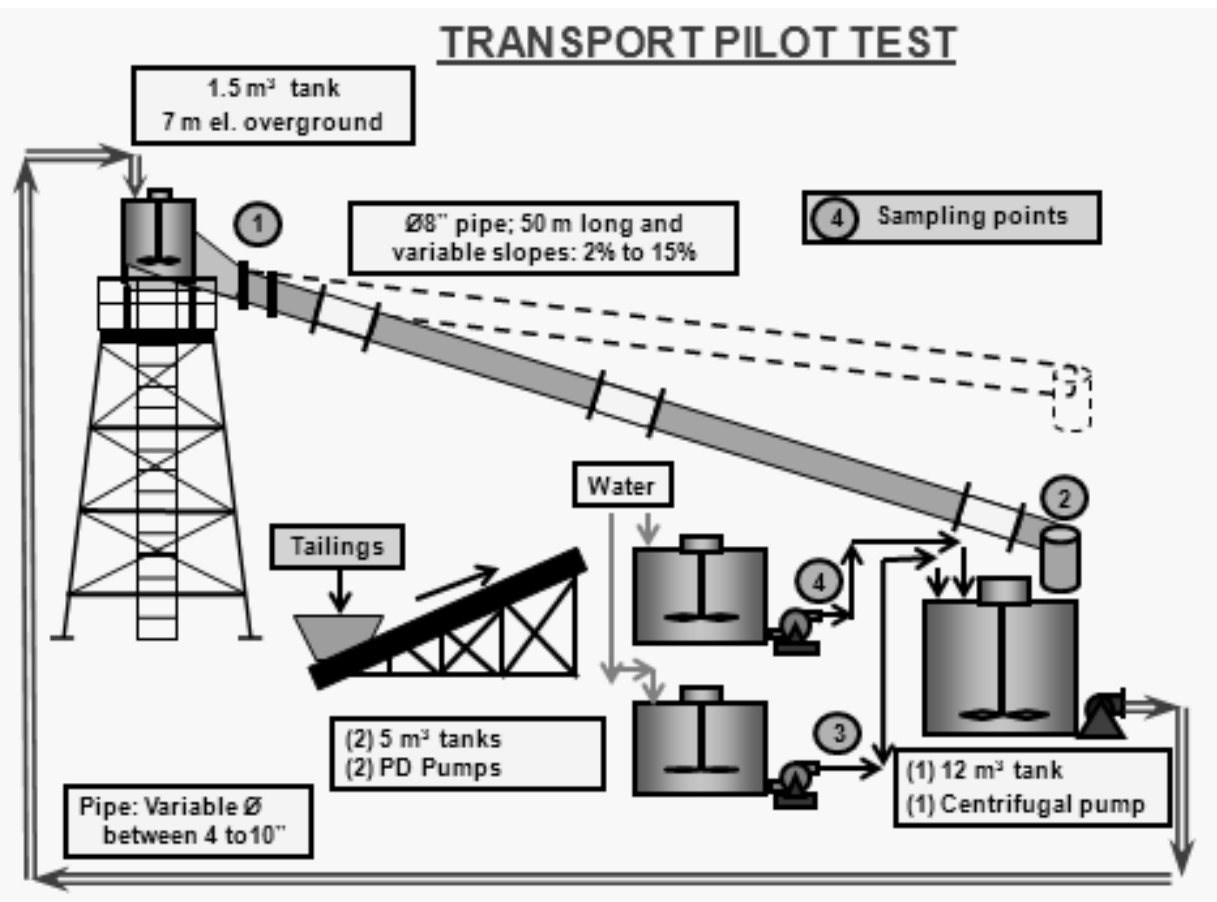

Figure 2 Transport pilot test diagram

The following main aspects are defined in the test:

- material

- geometry - cross section, length, and slope

- flow

- monitoring variables and location of measurement points

- duration of the test.

The duration of the test is determined as the minimum necessary to achieve the normal flow regime and take the specified number of measurements. The flow, length and type of tests define the minimum tailings volume necessary to perform the test. The slope depends on the objective of the test: verify the behaviour; geometrical similarity; validation of theoretical model; etc. The variables to monitor in the test are: height of the flow in locations 1 and 2, the flow (if possible by volume measurement) at the low end of the flume, and the temperature. In addition, before starting each test, samples of the tailings are taken to measure solids content, specific gravity, grain size, and yield stress at the laboratory.

One of the challenges consists in the preparation of the minimum volume of flocculated sample at the specified concentration. This preparation takes time because generally the flow required is much larger than the flow producing/discharging from the pilot thickener.

Among the more common difficulties in this type of tests is the sizing of pump motor, the motor-reducer of the agitators, the transfer of the slurries, the possible clogging of the pipes and pumps, the change in slope of the downcomer pipe/flume, etc. In one case it was necessary to more than double the power of the pump motor in order to achieve required velocity or flow rate (i.e. from 100-220 HP). This means a significant agility in quickly implementing (purchase or rent and installation) of the replacement.

\subsubsection{Under pressure transport tests}

One of the traditional ways of executing the pressure tests is in a loop in which all the elements are pipes under pressure. One of the problems with this type of tests in loop is the increase in temperature of the slurry during the test. This phenomenon alters the viscosity and, therefore, the variables to be measured. In certain cases, we have installed cooling equipment in the line to mitigate this effect. 
An alternative solution is that half of the loop be formed by open pipes that facilitate ventilation and the elimination of heat. This sometimes requires that the pipes respect the requirements of speed of the test. In this sense, equipment for testing in the flume is only usable for testing under pressure only if the measuring instruments are placed in the pipeline that goes from the pump to the elevated tank.

The following main aspects are defined in the under pressure test:

- material

- geometry — pipe diameter, length, and slope

- flow

- pump power

- monitoring variables and location of measurement points

- duration of the test.

The most common difficulties in this type of tests are similar to those in the open flow testing.

\subsection{Deposition tests}

The deposition tests have usually been performed in a flume (wooden, approximately $1 \mathrm{~m}$ in width and $10 \mathrm{~m}$ in length) or dug in the ground (Slottee et al., 2005) or lined with film (Yuan and Lahaie, 2009), where the tailings were discharged directly from the thickener at a very low flow and the inclination surface of the tailings was measured. It is our opinion that this test presents limitations because it does not take into account the energy of the discharge and runoff characteristics on the beach (meanderings) and it is not possible to extrapolate its results to real life. Recently a model (McPhail, 2008) was presented that relates the slope in the tailings beach with the energy of the jet of the tailings discharge. We understand that there are other models with the same objective but we have not had access to its theoretical formula for its execution or interpretation.

For the deposition tests based on the McPhail (2008) methodology, a test pad of $3.5 \mathrm{~m}$ in width by $10 \mathrm{~m}$ in length and $0.5 \mathrm{~m}$ in height is required. The tailings slurry is discharged at one end at a specified flow and height, both which must be maintained constant. Figure 3 presents a schematic diagram of the main equipment and monitoring locations of the test.

\section{DEPOSITION PILOT TEST}

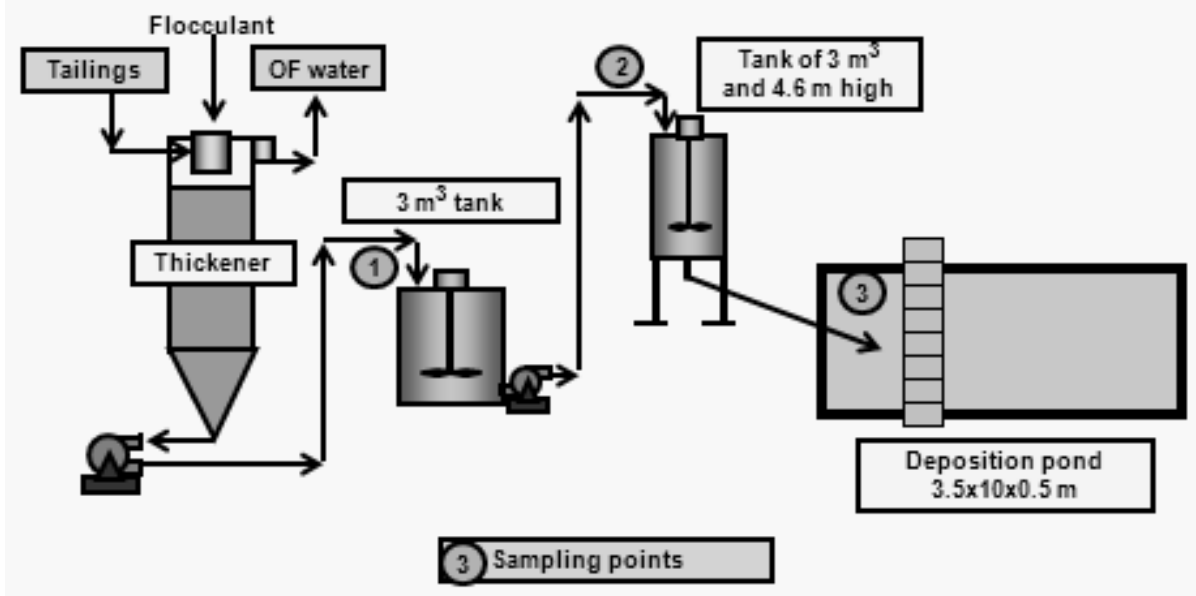

Figure 3 Deposition pilot test diagram 
The following main aspects are defined for the deposition test:

- accumulating tank with agitator of approximately $3 \mathrm{~m}^{3}$

- positive displacement pump for tailings transfer

- elevated tank of $4.6 \mathrm{~m}$ in height with agitator and $3 \mathrm{~m}^{3}$ volume

- bridge or beam $6 \mathrm{~m}$ in length for measurement and filming

- topographic devices to measure the level of the deposited tailings.

At the start of each test the following parameters are measured: the flow through direct volume measurement (volume versus time), solids content and geometric measurement of the discharge jet (width, distance of fall, height of fall, etc.). When the highest area of deposition reaches $30 \mathrm{~cm}$ in height, the previous measurements are repeated.

When the highest area of deposition reaches $40 \mathrm{~cm}$ in height, the deposition is finalised. After one hour a topographically survey of the longitudinal profile of the tailings beach is done. In addition, all the elements of the system are measured: height of tank, diameters, height of singularities (valves, breaks in slopes, etc.), length of pipes, hoses, etc. Samples are taken in the high and low areas of the beach for grain size tests and leave materials for other tests (rheology).

In case where the test is performed with flocculated tailings, the same challenge, already mentioned in the transport tests: preparation of the minimum volume of flocculated sample at the specific concentration occurs.

Another challenging aspect is assuring the constant flow which requires having a pair of tanks of adequate size and layout for this purpose.

Among the most common difficulties in these types of tests is the sizing of the motor-reducer of the agitators, the transfer of the thickened slurries, the possible clogging of the pipes and pump, etc.

\section{Conclusions}

The pilot tests applied to thickened tailings are a recognised tool to advance the knowledge of the behaviour of this material, in particular when the level of concentration nears that of paste.

For the execution of these tests, a proper preparation is required to reach the established objectives which includes the selection of representative material for the operation, the determination of the minimum tonnage required for the test, the selection of a behaviour model and parameters to be verified, the adoption of a test scale, the climate conditions (if relevant), etc. All these factors must be incorporated in the definition of the tests and in this manner define the characteristics of each test.

The examples presented are a way of approaching each case and present a large part of the problems and challenges that must be overcome to obtain results useful for the design and to advance in the knowledge of the behaviour of thickened tailings.

Bearing in mind that all pilot tests present a certain level of uncertainty in their behaviour, it is very important to allow sufficient duration of the tests program so that: (i) in theory the possible range of variables to be measured is verified; and (ii) the facility and equipment selected have the flexibility and availability to make the adjustments necessary to continue or perform the tests in real conditions.

\section{References}

Calle, F., González, A., Fernández, L. and Fuentes, R. (2009) Diseño de Sistemas de Transporte de Fluidos Bingham d Escala Industrial. Problemas de Scaling Up, XIX Congreso Chileno de Hidráulica, Sociedad Chilena de Ingeniería Hidráulica, Chile.

McPhail, G. (2008) Prediction of the Beach Profile of High Density Thickened Tailings from Rheological and Small Scale Trial Deposition Data, in Proceedings 11th International Seminar on Paste and Thickened Tailings (Paste08), A.B. Fourie, R.J. Jewell, P. Slatter and A. Paterson (eds), 5-9 May 2008, Kasane, Botswana, Australian Centre for Geomechanics, Perth, pp. 179-188. 
Slottee, S., Johnson, J. and Crozier, M. (2005) Paste Thickening Iron Ore Tailings, XXXV Ironmaking and Raw Materials Seminar, VI Brazilian Symposium on Iron Ore, Florianópolis, Santa Catarina, Brazil.

Yuan, X.S. and Lahaie, R. (2009) Thickened tailings (paste) technology and its applicability in oil sand tailings management, in Proceedings Thirteenth International Conference on Tailings and Mine Waste (Tailings and Mine Waste '09), Banff, Alberta, Canada, D. Sego, M. Alostaz, N. Beier (eds), University of Alberta Geotechnical Center, 1-4 November 2009, Edmonton, Alberta, pp. 829-1-829-16. 